\title{
Non-parametric counterfactual analysis in dynamic general equilibrium
}

Kubler, Felix ; Schmedders, Karl

\begin{abstract}
In this paper, we examine non-parametric restrictions on counterfactual analysis in a dynamic stochastic general equilibrium model. Under the assumption of time-separable expected utility and complete markets all equilibria in this model are stationary. The Arrow-Debreu prices uniquely reveal the probabilities and discount factor. The equilibrium correspondence, defined as the map from endowments to stationary (probability-free) state prices, is identical to the equilibrium correspondence in a standard Arrow-Debreu exchange economy with additively separable utility. We examine possible restriction on this correspondence and give necessary as well as sufficient conditions on profiles of individual endowments that ensure that associated equilibrium prices cannot be arbitrary. Although restrictions on possible price changes often exist, we show that results from a representative-agent economy usually do not carry over to a setting with heterogeneous agents.
\end{abstract}

DOI: https://doi.org/10.1007/s00199-009-0475-8

Posted at the Zurich Open Repository and Archive, University of Zurich

ZORA URL: https://doi.org/10.5167/uzh-27210

Journal Article

Published Version

Originally published at:

Kubler, Felix; Schmedders, Karl (2010). Non-parametric counterfactual analysis in dynamic general equilibrium. Economic Theory, 45(1-2):181-200.

DOI: https://doi.org/10.1007/s00199-009-0475-8 


\title{
Non-parametric counterfactual analysis in dynamic general equilibrium
}

\author{
Felix Kubler • Karl Schmedders
}

Received: 22 July 2008 / Accepted: 26 May 2009 / Published online: 19 June 2009

(C) Springer-Verlag 2009

\begin{abstract}
In this paper, we examine non-parametric restrictions on counterfactual analysis in a dynamic stochastic general equilibrium model. Under the assumption of time-separable expected utility and complete markets all equilibria in this model are stationary. The Arrow-Debreu prices uniquely reveal the probabilities and discount factor. The equilibrium correspondence, defined as the map from endowments to stationary (probability-free) state prices, is identical to the equilibrium correspondence in a standard Arrow-Debreu exchange economy with additively separable utility. We examine possible restriction on this correspondence and give necessary as well as sufficient conditions on profiles of individual endowments that ensure that associated equilibrium prices cannot be arbitrary. Although restrictions on possible price changes often exist, we show that results from a representative-agent economy usually do not carry over to a setting with heterogeneous agents.
\end{abstract}

\footnotetext{
We gratefully acknowledge support from the Swiss Finance Institute. We thank an associate editor and two anonymous referees for helpful comments on an earlier version and Don Brown and Jan Werner as well as seminar participants at the 2007 SAET conference in Kos/Greece and at Georgetown University for helpful discussions.
}

\section{F. Kubler}

Swiss Banking Institute, University of Zurich, Zurich, Switzerland

e-mail: kubler@isb.uzh.ch

F. Kubler $\cdot$ K. Schmedders

Swiss Finance Institute, Zurich, Switzerland

K. Schmedders $(\varangle)$

Institute for Operations Research, University of Zurich, Zurich, Switzerland

e-mail: karl.schmedders@ior.uzh.ch; k-schmedders@kellogg.northwestern.edu 
Keywords Dynamic general equilibrium $\cdot$ Non-parametric analysis $\cdot$ Observable restrictions

JEL Classification $\mathrm{D} 50 \cdot \mathrm{G} 10$

\section{Introduction}

This paper investigates how equilibrium prices may change as profiles of endowments vary in a dynamic asset pricing model with heterogeneous agents. In this model all competitive equilibria are stationary if all agents maximize time-separable expected utility and individual endowments follow time-homogeneous Markov chains. In fact, there is a one-to-one relation between the equilibrium correspondence in this model and the equilibrium correspondence of a standard static Arrow-Debreu exchange economy with additively separable utility. We use the non-parametric analysis of Brown and Matzkin (1996) to explore observable restrictions on this equilibrium correspondence. The three main results are as follows. First, we show that equilibrium price changes can be arbitrary if individual endowments vary but aggregate endowments are held fixed. Second, we show that changes in aggregate endowments always lead to 'predictable' price changes, if in at least one state aggregate endowments weakly decrease while in some other state all individuals' endowments increase. Last, we show that restrictions from the representative-agent version of the model are neither necessary nor sufficient for restrictions in a heterogeneous agents economy.

Dynamic general equilibrium models play a prominent role in modern macroeconomics, finance, and public finance. While stochastic dynamic models with heterogeneous agents have become increasingly important in this literature, there are few general results on counterfactual analysis. ${ }^{1}$ In applied work, computational experiments are often successfully employed to explore the effects of exogenous changes in taxes or transfers on equilibrium allocations and prices. In this literature all individuals in the economy are often assumed to have identical homothetic utility, but the computational results appear relatively robust with respect to small changes in preferences. Many results in this literature depend crucially on the exact parametric specification of preferences and, from a theoretical viewpoint, it might appear that without any assumptions on preferences almost any counterfactual analysis could be possible. While Brown and Matzkin (1996) successfully challenge the view that without parametric assumptions on preferences 'anything goes' in general equilibrium analysis, there have been few attempts in the literature to characterize the exact form of the observable restrictions and there are no results for infinite horizon stochastic economies frequently used in applied work. This paper addresses this void in the economic literature.

\footnotetext{
1 The use of the term "counterfactual analysis" in this paper is inspired by the usage of the term "counterfactual policy analysis" in macroeconomics. We use this term in the sense of a global comparative statics analysis and want to distinguish our analysis from purely local comparative statics or marginal analysis, often used in consumer and producer theory.
} 
For the investigation of counterfactual analysis in a dynamic model with heterogeneous agents we consider the simplest possible case and ask what happens to equilibrium prices as profiles of individual endowments change. We regard the consideration of changes in individual endowments as a first step towards understanding the more complicated comparative statics exercises arising in applied work.

We consider a version of the Lucas (1978) asset pricing model with heterogeneous investors. All exogenous variables follow a finite Markov chain and all individuals maximize time-separable expected utility with identical beliefs and discounting. When markets are dynamically complete, all asset market equilibria are equivalent to ArrowDebreu equilibria with stationary consumption allocations (see e.g., Kubler and Schmedders 2003). We show that the price system reveals uniquely the agents' beliefs and discount factor. If there are $S$ possible shocks, the prices can, in fact, be decomposed into the $S \times S$ transition probability matrix, a discount factor and $S$ strictly positive 'probability-free prices' that are unique up to a normalization. The question is then what restrictions exist on the changes of these probability-free prices.

The results depend to some extent on the preference assumptions. The assumption of time-separable expected utility leaves open the possibility that felicity functions are shock-dependent. Melino and Yang (2003) and Danthine et al. (2004) emphasize the usefulness of this assumption for explaining standard asset pricing puzzles. The focus of this paper is on economies where utility functions can be shock dependent. In a working-paper version (Kubler and Schmedders 2007) we compare the results to the case of state-independent utility. It is not easily possible to extend the analysis to the case of Epstein-Zin or recursive utility since under this specification Pareto-efficient allocations are generally no longer stationary (see e.g., Dumas et al. 2000). Kubler (2003) examines the case for finite non-stationary economies and shows that there are some restrictions under recursive utility.

Under our assumption of shock-dependent felicity functions, restrictions on prices exist-even in the single-agent framework-only if in at least one shock aggregate endowments decrease while in some other they increase. With several agents, changes in the income distribution with aggregate endowments held fixed can have arbitrary effects on equilibrium prices. These results have potentially important implications for applied modeling. Without parametric assumptions on preferences, there are no restrictions on price changes resulting from a change in the wealth distribution, i.e., the introduction of purely redistributive taxes can have arbitrary effects. However, we also show that if all agents' individual endowments increase in one shock, while aggregate endowments weakly decrease in some other shock, the associated prices cannot be arbitrary. This condition is in line with the intuition that if in some state aggregate endowments decrease while in some other they increase, prices must change in a predictable way. If applied work considers policy experiments that have aggregate effects, the resulting price changes cannot be arbitrary. These results also raise the question how the restrictions in a model with several agents that arise through changes in aggregate endowments are related to restriction in the representative-agent model.

In models with complete financial markets, under the assumption that all agents maximize time-separable expected utility, there exists a pricing representative agent: Any given competitive equilibrium price system can be viewed as supporting prices 
for a single individual who consumes aggregate endowments (see e.g., Constantinides 1982). However, obviously, this fact does not imply that in models with heterogeneous agents, results from counterfactual analysis are similar to the ones in a model with a single agent. In this paper, we investigate if there are any restrictions on global comparative statics in models with several agents and how they compare to the single-agent intuition. We show that there exist changes in endowments for which there exist restrictions on associated prices in a model with several agents even if there are no restrictions in the single-agent model, given the changes in aggregate endowments. Conversely, as already noted above, the existence of restrictions in a single-agent economy does not imply restrictions in economies with several agents, if individual endowments can be chosen freely. Even if individual endowments are collinear, the qualitative predictions from the heterogeneous agent model are very different from the ones in the single agent economy.

The remainder of the paper is organized as follows. In Sect. 2, we introduce the model and show its equivalence to a static Arrow-Debreu model with additively separable utility. In Sect. 3, we motivate why we are interested in counterfactual analysis and show how in this model beliefs can be identified separately from prices. Section 4 presents necessary as well as sufficient conditions on changes in endowments for equilibrium price changes not to be arbitrary. In Sect. 5, we compare the restriction of the model with several agents to restrictions that arise in the single-agent framework. Section 6 concludes.

\section{The dynamic exchange economy}

We consider a dynamic exchange economy under uncertainty with a single perishable good each period. Time is discrete, $t=0,1, \ldots, T \leq \infty$. Uncertainty is driven by exogenous shocks $s_{t}$ that take values in a finite set $\mathcal{S}=\{1,2, \ldots, S\}$ and follow a Markov chain with transition matrix $\pi$. Without loss of generality we assume that $s_{0}=1$. A date-event is a finite history of shocks, $\sigma=s^{t}=\left(s_{0}, s_{1}, \ldots, s_{t}\right)$ and the set of all date-events (nodes) of the event tree is denoted by $\Sigma$. We write $\sigma$ for a generic node in the tree and collect all nodes at some time $t$ in $\mathcal{N}_{t}=\left\{s^{t}\right\}$. We write $\pi\left(s^{t}\right)$ for the period zero probability of node $s^{t}$ and $\pi\left(s_{t-1}, s_{t}\right)$ for the one-period transition probabilities, that is, the elements of the matrix $\pi$.

There are $H$ types of $T$-period (possibly infinitely) lived agents, $h \in \mathcal{H}=$ $\{1,2, \ldots, H\}$. Individual endowments are a time-invariant function of the exogenous shock alone, $e^{h}\left(s^{t}\right)=\bar{e}^{h}\left(s_{t}\right)$ with $\bar{e}^{h}: \mathcal{S} \rightarrow \mathbb{R}_{++}$. Agents have time-separable expected utility (EU) preferences represented by the utility function

$$
U^{h}(c)=E_{0} \sum_{t=0}^{T} \beta^{t} u^{h}\left(c\left(s^{t}\right), s_{t}\right),
$$

where $\beta \in(0,1)$, and for each $s \in \mathcal{S}, u^{h}(\cdot, s): \mathbb{R}_{+} \rightarrow \mathbb{R}$ is strictly concave, strictly increasing and continuous for $c>0$. 


\subsection{Arrow-Debreu equilibrium}

In this paper, we abstract from asset markets and simply assume that markets for commodities across all nodes of the event tree are complete (see Kubler and Schmedders 2003 for a formal analysis of the model with asset markets). We can therefore describe the resulting Arrow-Debreu economy simply as a collection of utility functions and individual endowments $\left(U^{h}, e^{h}\right)^{h \in \mathcal{H}}$. An Arrow-Debreu equilibrium for this economy is defined in standard fashion as a collection of state prices $p(\sigma)>0$ and consumption allocations $c^{h}(\sigma) \geq 0$ for all nodes $\sigma \in \Sigma$ such that

1. Markets clear, $\sum_{h \in \mathcal{H}}\left(c^{h}(\sigma)-e^{h}(\sigma)\right)=0$ for all $\sigma \in \Sigma$;

2. Agents optimize, $\forall h \in \mathcal{H}\left(c^{h}(\sigma)\right)_{\sigma \in \Sigma} \in \arg \max U^{h}(c)$ s. t.

$$
\sum_{\sigma \in \Sigma} p(\sigma) c(\sigma)=\sum_{\sigma \in \Sigma} p(\sigma) e^{h}(\sigma)<\infty .
$$

Arrow-Debreu equilibrium exists and both the first and second welfare theorem hold for these economies (Bewley 1972). The following lemma provides the key to tractable computations in stationary models with many periods. The lemma is well known, see for example the textbook by Duffie (1988) or Kubler and Schmedders (2003).

Lemma 1 Given an efficient allocation $\left(c^{h}(\sigma)\right)_{\sigma \in \Sigma}^{h \in \mathcal{H}}$, the individual consumptions must be time-invariant functions of the shock alone, i.e., there exist $\bar{c}: \mathcal{S} \rightarrow \mathbb{R}_{+}^{H}$ such that for all $s^{t} \in \Sigma$ and all $h \in \mathcal{H}, c^{h}\left(s^{t}\right)=\bar{c}^{h}\left(s_{t}\right)$.

Kubler and Schmedders (2003) show how the model can be reformulated as a model of a "stochastic finance economy" with stock and bond markets (i.e., a Lucas tree economy with heterogeneous agents but dynamically complete markets) and prove for generic asset payoffs that the Arrow-Debreu and the finance model are equivalent. We focus on the Arrow-Debreu equilibrium in this paper but most of our results extend to the equivalent model.

The lemma implies that the Euler equations show that for any Arrow-Debreu equilibrium prices $\left(p\left(s^{t}\right)\right)$, the expression $p\left(s^{t}\right) /\left(\beta^{t} \pi\left(s^{t}\right)\right)$ just depends on the current shock $s_{t}$ but not on the history. We can therefore define stationary probability-free prices by $\rho\left(s_{t}\right) \equiv \frac{p\left(s^{t}\right)}{\pi\left(s^{t}\right) \beta^{t}}$ for all $s_{t} \in \mathcal{S}$. Based on this definition we next define the equilibrium correspondence as the map $\omega: \mathbb{R}_{++}^{H S} \rightarrow \mathbb{R}_{++}^{S}$ from $H S$-dimensional profiles of endowments to $S$-dimensional equilibrium prices $\rho=(\rho(s))_{s \in \mathcal{S}}$ by

$$
\omega\left(e^{h}\right)=\left\{\rho \in \mathbb{R}_{++}^{S}:\left(\rho\left(s_{t}\right) \beta^{t} \pi\left(s^{t}\right)\right)_{s^{t} \in \Sigma}\right. \text { are Arrow-Debreu prices }
$$

$$
\text { for the economy with endowments } \left.e^{h}\right\} \text {. }
$$

\subsection{Reduction to finitely many goods}

We can impose the fact that each agent makes stationary consumption choices in any Arrow-Debreu equilibrium directly in the budget constraints. Therefore, we 
can reformulate an agent's budget constraint $\sum_{\sigma \in \Sigma} p(\sigma)\left(c(\sigma)-e^{h}(\sigma)\right)=0$ as follows:

$$
\rho\left(s_{0}\right)\left(c\left(s_{0}\right)-e\left(s_{0}\right)\right)+\sum_{t=1}^{T} \beta^{t} \sum_{s^{t} \in \mathcal{N}_{t}} \pi\left(s^{t}\right) \rho\left(s_{t}\right)\left(c\left(s_{t}\right)-e\left(s_{t}\right)\right)=0 .
$$

Note that consumptions and endowments at time $t$ only depend on the current shock $s_{t}$ and not on any part of the history before $t$. For $t=2, \ldots, T$ define (recursively) the $t$-fold product of the Markov transition matrix, $\pi^{t}=\pi \cdot \pi^{t-1}$. Observe that the probabilities in the first row of $\pi^{t}$ yield the distribution for the current state in period $t$ since we assumed w.l.o.g. that the economy starts in state $s_{0}=1$. Next define $\Phi=\sum_{t=0}^{T} \beta^{t} \pi^{t}$ and let $\phi=\left(\phi_{1}, \ldots, \phi_{S}\right)$ denote the first row of the matrix $\Phi$. We sometimes write $\phi(\beta, \pi)$ to make explicit the dependence on the discount factor and the transition probabilities.

Each agent's utility maximization problem, being reduced to stationary consumption choices, can now be written as

$$
\max \sum_{s=1}^{S} \phi_{s} u^{h}(c(s), s) \quad \text { subject to } \sum_{s=1}^{S} \phi_{s} \rho(s)(c(s)-e(s))=0 \text {. }
$$

Observe that an equilibrium is now a vector of prices $\rho \in \mathbb{R}_{++}^{S}$ and choices $c^{h} \in \mathbb{R}_{+}^{S}$ for all $h \in \mathcal{H}$ such that each agent maximizes utility and markets clear. This observation implies that any restriction on the equilibrium correspondence of a Walrasian model (i.e., an Arrow-Debreu model with finitely many commodities) with separable utility translates one to one, to restrictions of the intertemporal model (prices are just the Walrasian prices, multiplied by $\phi_{s}$ and vice versa). The following notation proves useful: $\phi \circ \rho=\left(\phi_{1} \rho(1), \ldots, \phi_{S} \rho(S)\right)$.

\section{Non-parametric counterfactual analysis}

The purpose of this paper is to examine the possible changes in Walrasian equilibrium prices that may result when agents' individual endowments vary. Our objective is to perform this examination in much generality. For this purpose we do not choose particular functional forms for utility functions but instead rely on a non-parametric approach along the lines of the methodology in the seminal paper by Brown and Matzkin (1996). Brown and Matzkin use the so-called Afriat inequalities (Afriat 1967) to examine whether observations on individual endowments and prices yield restrictions on the Walrasian equilibrium correspondence. In this paper we use the Afriat inequalities to analyze global comparative statics for the dynamic model of the previous section.

Section 3.1 introduces and motivates the non-parametric approach to counterfactual analysis. We often maintain the language of the literature started by Brown and Matzkin (1996) even though it may sound unusual at times for a comparative statics analysis. For example, we adopt the term 'observations' to refer to different specifications of endowments together with equilibrium prices. We argue below how our 
analysis can be interpreted within the methodological framework laid out by Brown and Matzkin (1996) but also give an alternative interpretation. Section 3.2 develops the Afriat inequalities for our model. Lemma 3 states simplified necessary and sufficient conditions for the inequalities to hold. Section 3.3 completes the development of our framework with the statement of Lemma 4, a special version of the main result of Brown and Matzkin (1996) for our model. The lemma provides necessary and sufficient conditions for prices to occur in a Walrasian equilibrium for given individual endowments.

\subsection{A set-up for global comparative statics}

We consider $N$ profiles of individual endowments $e^{h}(i)$ for $h \in \mathcal{H}$ and $i \in \mathcal{E}=$ $\{1,2, \ldots, N\}$ with $e^{h}(i)=\left(e_{1}^{h}(i), \ldots, e_{S}^{h}(i)\right) \in \mathbb{R}_{++}^{S}$ and say that associated prices $\left(p_{\sigma}(i)\right)_{i \in \mathcal{E}, \sigma \in \Sigma}$ are consistent with equilibrium if there are $(\rho(i))_{i \in \mathcal{E}}, \beta \in(0,1)$ and a Markov transition matrix $\pi$ with

$$
\rho\left(s_{t}\right)=\frac{p\left(s^{t}\right)}{\pi\left(s^{t}\right) \beta^{t}} \quad \text { for all } s_{t} \in \mathcal{S},
$$

and if there is an equilibrium correspondence, $\omega$, such that

$$
\rho(i) \in \omega\left(\left(e^{h}(i)\right)^{h \in \mathcal{H}}\right) \quad \text { for all } i \in \mathcal{E} \text {. }
$$

We sometimes refer to $\left(\left(e^{h}(i)\right)^{h \in \mathcal{H}}, p(i)\right)_{i \in \mathcal{E}}$ as 'observations'. Since we do not require endowments to be different across specifications, we trivially make statements about the equilibrium set of a given economy.

We ask which price changes are consistent with changes in the profile of individual endowments. For our model with a large and possible infinite event tree examining the vector (for finite $T$ ) or the sequence (for $T=\infty$ ) of such prices may at first appear rather daunting. However, the assumption that the observations are generated by an Arrow-Debreu equilibrium of the underlying dynamic exchange economy puts a lot of structure on the equilibrium prices. In particular, we can prove that the Arrow-Debreu prices can be uniquely decomposed into transition probabilities $\pi$, the discount factor $\beta$ and probability free prices $\rho$.

\subsubsection{Beliefs versus prices}

The absence of arbitrage and the stationarity of the equilibrium allocations implies a one-to-one relation between Arrow-Debreu prices, $(p(\sigma))_{\sigma \in \Sigma}$, and the prices of 'one-period Arrow securities'. Let $a_{s, s^{\prime}}=p\left(s^{t+1}\right) / p\left(s^{t}\right)$ whenever $s_{t+1}=s^{\prime}$ and $s_{t}=s$, that is, $a_{s, s^{\prime}}$ is the price of a one-period security that pays one unit of the consumption good in the next period if state $s^{\prime}$ occurs and nothing in all other states. Let $A=\left(a_{s, s^{\prime}}\right)_{s, s^{\prime}=1}^{S}$ be the $S \times S$ matrix of the prices of all such one-period Arrow securities. Theorem 1 states if these prices are equilibrium prices, then the underlying transition probabilities and the discount factor can be recovered uniquely. 
Theorem 1 For a given matrix of one-period Arrow security prices $A$, there exists a unique Markov transition matrix $\pi$, a unique discount factor $0<\beta<1$ and prices $\rho \in \mathbb{R}_{++}^{S}$, unique up to a normalization, such that for all $s, s^{\prime} \in \mathcal{S}$,

$$
a_{s, s^{\prime}}=\beta \pi\left(s, s^{\prime}\right) \frac{\rho_{s^{\prime}}}{\rho_{s}}
$$

Proof We prove that for a given positive matrix $A$ the nonlinear system of $S^{2}$ equations

$$
a_{s, s^{\prime}} \frac{\rho_{s}}{\rho_{s^{\prime}}}=\beta \pi\left(s, s^{\prime}\right)
$$

in the unknown discount factor $\beta$, transition matrix $\pi$, and probability-free prices $\rho$ has a unique positive solution. Summing all equations for fixed $s$ and using the property of $\pi$ that all row elements sum to 1 we obtain

$$
\sum_{s^{\prime}=1}^{S} a_{s, s^{\prime}} \frac{\rho_{s}}{\rho_{s^{\prime}}}=\beta \quad \text { for all } s \in \mathcal{S} \text {. }
$$

Defining $\gamma_{s}=1 / \rho_{s}$ and $\gamma=\left(\gamma_{1}, \ldots, \gamma_{S}\right)^{\top}$, we obtain the linear system of equations

$$
\left(A-\beta I_{S \times S}\right) \gamma=0
$$

where $I_{S \times S}$ denotes the $S \times S$ identity matrix. Note that this system of linear equations is just the system defining the eigenvalues and eigenvectors of the matrix $A$ ! The classical Perron-Frobenius theorem (see e.g., Horn and Johnson 1985) implies that the positive matrix $A$ has a unique largest real eigenvalue, $\beta^{*}$, that is positive and associated with a positive real eigenvector, $\gamma^{*}$. Furthermore all other eigenvalues are associated with eigenvectors that are not non-negative. Therefore, there is only one solution for $\beta>0$ with associated $\gamma>0$. Since the elements of the matrix $A$ are generated by our dynamic exchange economy this unique solution must also satisfy $\beta<1$. Furthermore, note that $\rho_{s} / \rho_{s^{\prime}}=\gamma_{s^{\prime}} / \gamma_{s}$ and so the prices $\rho$ are also uniquelyup to a normalization - determined which finally leads to a unique transition matrix $\pi$.

The equilibrium conditions of our dynamic exchange economy immediately imply that for a given discount factor $\beta$, a given transition matrix $\pi$, and probabilityfree prices $\rho$ there exist uniquely determined Arrow-Debreu equilibrium prices. Theorem 1 establishes the converse of this property. From an observation of ArrowDebreu equilibrium prices we can recover the transition matrix and agents' discount factor in addition to probability-free state prices. This recoverability of the transition matrix and the discount factor has an important consequence for our analysis in this paper. We do not need to make a case distinction depending on whether (or not) we know the transition probabilities and discount factors. Given the assumption of the observability of Arrow-Debreu prices we can immediately assume that we know $\beta$ and $\pi$. 
The result of Theorem 1 resembles the work of Wang (1993), Cuoco and Zapatero (2000) and others on the recoverability problem of preferences and beliefs in a continuous-time infinite-horizon economy with dynamically complete financial markets. The methods in this literature are very different from our application of the Perron-Frobenius theorem here. For example, the analysis of Markovian equilibria in a continuous-time setting requires (Cuoco and Zapatero 2000) to examine a Riccati differential equation.

\subsubsection{Interpretation}

Based on Theorem 1 restrictions on global comparative statics can always be viewed in two parts. First, different price systems across multiple observations must reveal the same beliefs and discount factor. Second, only the probability-free prices can change across multiple observations. We say that there exist restrictions on prices, if, given $N$ profiles of endowments $\left(e^{h}(i)\right)_{i \in \mathcal{E}}^{h \in \mathcal{H}}$, there exist $(\rho(i))_{i \in \mathcal{E}} \gg 0$ that are not consistent with equilibrium. For the investigation of restrictions on the probability-free prices, we consider restrictions on the equilibrium correspondence $\omega$ as defined in Sect. 2.1. In the following, we always assume that prices are strictly positive and that endowments are nonnegative.

The restrictions on the equilibrium correspondence of the dynamic model must be identical to those of the standard Walrasian model when utility is separable. For general non-separable utility, these restrictions have first been investigated by Brown and Matzkin (1996) who give an example to show that some restrictions exist. Brown and Matzkin interpret their exercise in terms of refutability of the general equilibrium model. They give necessary and sufficient conditions on the equilibrium correspondence and reserve the term 'comparative statics' for necessary conditions only.

In a dynamic general equilibrium model, what is observable is generally only one equilibrium. Testable restrictions on possible equilibrium prices then come from assumptions on preferences (over and above expected time-separable utility, see Kubler (2003)). Here we examine changes in prices as endowments vary, i.e., we consider equilibria of different economies.

We interpret this endowment change as the result of an unanticipated policy change to which all agents in the economy assign probability zero. If at some node $s^{t}$, a transfer and lump-sum tax scheme is introduced without agents in the economy anticipating so, equilibrium prices are going to adjust instantly to the new equilibrium corresponding to different endowments. This is the classic exercise of counterfactual policy analysis which is standard in public finance and macro. Researchers fix functional forms for utility and production functions and use numerical methods to compute equilibrium for different specifications of the exogenous variables. Here we ask the fundamental question if anything can be said without fixing functional forms. We examine a simpler model and simpler comparative statics than commonly used in applications but do so under very general assumptions without using parametric functional forms. We thus view our results as a first step towards a general non-parametric policy analysis.

Alternatively, and more in line with Brown and Matzkin (1996) original approach one can interpret the exercise as a 'structural break' and interpret the results as 
'observable restrictions'. Either all agents attach zero probability to this structural break or else it is completely uninsurable, that is, no asset pays contingent on this shock to the economy occurring. If there are Lucas trees or other multi-period assets in the economy, it is a very strong assumption that the structural break is not insurable, since it amounts to saying that in that state, all trees become worthless, that is, they no longer pay any dividends from there on. The presence of Lucas trees also makes the interpretation of an unanticipated change more complicated. The new endowment profiles now consist of the actual individual endowments plus the dividends of the Lucas trees an individual held at the time of the change. It is subject to further research to explore this interpretation in more detail.

\subsection{Individual Afriat inequalities}

For the characterization of competitive equilibria Brown and Matzkin (1996) use the Afriat inequalities and examine if observed aggregate demand can be expressed as the sum of (unknown) individual choices which satisfy those inequalities and a budget constraint. Brown and Matzkin (1996) give an example showing that these conditions are not vacuous. In a simple model with two agents and two commodities, it is possible to find variations of endowments and prices that are inconsistent with equilibrium. In our framework we need to consider slightly different conditions, since utility is additively separable across states. This fact implies that the conditions of Brown and Matzkin remain necessary but are no longer sufficient.

Afriat (1967) formulates a system of linear inequalities which characterize a finite set of observations of individual choices arising from utility maximization. His techniques can be applied to a wide variety of frameworks. For example, Afriat inequalities can characterize optimal asset demand, savings and demand of goods, see Varian (1983a,b). The basic ideas are to assume that the utility function is strictly concave and continuous, to use the Kuhn-Tucker theorem to characterize optimality, to relate the subgradient of the utility function to prices, and to characterize concavity in terms of the subgradients being negatively co-monotone to consumption. The following lemma states the Afriat inequalities:

Lemma 2 Given $\phi \in \mathbb{R}_{++}^{S}$ as well as consumptions and prices $(c(i), \rho(i))_{i \in \mathcal{E}}$, the following two statements are equivalent:

1. There exists a utility function with strictly increasing, strictly concave and continuous $u(c, s), s \in \mathcal{S}$, such that for all $i \in \mathcal{E}$

$$
c(i) \in \arg \max _{c \in \mathbb{R}_{+}^{S}} \sum_{s=1}^{S} \phi_{s} u\left(c_{s}, s\right) \text { s.t. }(\phi \circ \rho(i)) \cdot(c-c(i)) \leq 0 .
$$

2. Consumptions and prices $(c(i), \rho(i))_{i \in \mathcal{E}}$ satisfy the following 'Afriat inequalities'. There exist $(\lambda(i))_{i \in \mathcal{E}} \gg 0$ such that for any $s \in \mathcal{S}$ and all $i, j \in \mathcal{E}$

$$
\left(c_{S}(i)-c_{S}(j)\right)\left(\lambda(i) \rho_{S}(i)-\lambda(j) \rho_{s}(j)\right) \leq 0,
$$


with strict inequality if $c_{S}(i) \neq c_{S}(j)$ and with $\lambda(i) \rho_{s}(i)=\lambda(j) \rho_{s}(j)$ if $c_{S}(i)=$ $c_{S}(j)>0$.

Proof 1. $\Rightarrow$ 2. The claim follows from convex analysis, see Rockafellar (1970). For all $s \in \mathcal{S}$ the function $u(\cdot, s)$ has a nonempty subdifferential $\partial_{c_{s}} u\left(c_{s}, s\right)$ and $v_{s}>0$ for all subgradients $v_{s} \in \partial_{c_{s}} u\left(c_{s}, s\right)$. Optimality of $c(i)$ implies that there exist $v_{s}(i) \in \partial_{c_{s}} u\left(c_{s}(i), s\right)$ as well as $\lambda(i)>0$ such that $\phi_{s} v_{s}(i)-\lambda(i) \phi_{s} \rho_{s}(i) \leq 0$ and equal to zero if $c_{s}(i)>0$. Strict concavity of each $u(\cdot, s)$ and $c_{S}(i)>c_{S}(j)$ implies $v_{s}(i)<v_{s}(j)$ and thus $\lambda(i) \rho_{s}(i)<\lambda(j) \rho_{s}(j)$. Note that $c_{s}(i)=c_{s}(j)>0$ immediately implies $\lambda(i) \rho_{s}(i)=\lambda(j) \rho_{s}(j)$. Now the Afriat inequalities follow.

2 . $\Rightarrow 1$. Assume without loss of generality that $c_{S}(1) \leq c_{S}(2) \leq \cdots \leq c_{S}(N)$. Define a positive and strictly decreasing piecewise linear function by setting $u^{\prime}\left(c_{s}(i), s\right)=\lambda(i) \rho_{s}(i)$ for $c_{s}(i)>0$ and $u^{\prime}(0, s)=1+\max _{j} \lambda(j) \rho_{s}(j)$. Moreover, for $c>0$ with $c_{s}(i)<c<c_{s}(i+1)$ let $u^{\prime}(c, s)=u^{\prime}\left(c_{s}(i), s\right)+\frac{c-c_{s}(i)}{c_{s}(i+1)-c_{s}(i)}\left(u^{\prime}\left(c_{s}(i+\right.\right.$ $1), s)-u^{\prime}\left(c_{s}(i), s\right)$ ) (including the special case $c_{s}(0)=0$ if $\left.c_{s}(1)>0\right)$. The constructed marginal utility functions are positive and strictly decreasing and thus integrate to strictly increasing and strictly concave utility functions on the respective intervals $[0, k)$ for $k>c_{S}(N)$. Observe that by construction the necessary and sufficient firstorder conditions for the utility maximization problem are satisfied.

Unlike the case of general utility functions, where versions of the strong axiom of revealed preferences (e.g., GARP) provide an alternative characterization, no equivalent conditions that are quantifier-free are known for additively separable utility functions. Varian (1983a) discusses various specifications for which GARP-like restrictions can be derived, but for the case of additively separable utility, he concludes "I have been unable to find a convenient combinatorial condition that is necessary and sufficient for additive separability". While we cannot give conditions that are simultaneously necessary and sufficient we can state simple necessary as well as sufficient conditions for the Afriat inequalities in the presence of additively separable utility functions.

The necessary condition considers the case where in one shock consumption increases while in some other it weakly decreases. In this situation the supporting price of the first shock must decrease relative to the price of the second shock. The sufficient condition considers the case of consumption increasing in all shocks, that is, there is no shock where consumption weakly decreases. In this case there are no restrictions on supporting prices.

Lemma 3 Necessary and sufficient conditions for consumption and price vectors $(c(i), \rho(i))_{i \in \mathcal{E}}$ to satisfy the Afriat inequalities are as follows:

(N) If $c_{S}(i)>c_{S}(j)$ then the Afriat inequalities imply for all $i, j \in \mathcal{E}$ and all $s^{\prime} \neq s$,

$$
c_{s^{\prime}}(i) \leq c_{s^{\prime}}(j) \Longrightarrow \frac{\rho_{s^{\prime}}(i) / \rho_{S}(i)}{\rho_{s^{\prime}}(j) / \rho_{s}(j)}>1 .
$$

(S) Iffor all $i, j, c(i) \gg c(j)$ (or vice versa), then consumption vectors $(c(i))_{i \in \mathcal{E}} \geq 0$ and any arbitrary price vectors $(\rho(i))_{i \in \mathcal{E}} \gg 0$ satisfy the Afriat inequalities. 
Proof For the proof of the sufficient conditions suppose without loss of generality that $c(1) \ll c(2) \ll \cdots \ll c(N)$. Observe that it is possible to choose $\lambda(1)>\lambda(2)>$ $\cdots>\lambda(N)$ both sufficiently large and different such that $\lambda(i) \rho(i) \gg \lambda(i+1) \rho(i+1)$ for all $i=1, \ldots, N-1$ and so that conditions (1) in statement 2 of Lemma 2 are satisfied.

Conversely, conditions (1) imply if $c_{S}(i)>c_{S}(j)$ for some $s \in \mathcal{S}$ that $0<$ $\lambda(i) \rho_{s}(i)<\lambda(j) \rho_{s}(j)$. Similarly, $c_{s^{\prime}}(i) \leq c_{s^{\prime}}(j)$ implies $\lambda(i) \rho_{s^{\prime}}(i) \geq \lambda(j) \rho_{s^{\prime}}(j) \geq$ 0 . Dividing the second weak inequality by the first strict inequality then yields the inequality of the lemma.

Lemma 3 is important for most of our arguments below. Although the lemma appears simple, especially the sufficiency part turns out to be applicable to a variety of situations.

\subsection{Equilibrium}

The following lemma specializes the key result of Brown and Matzkin (1996) to the case of additively separable utility.

Lemma 4 (Brown and Matzkin 1996) Observations on prices and individual endowments $\left(\rho(i),\left(e^{h}(i)\right)^{h \in \mathcal{H}}\right)_{i \in \mathcal{E}}$, are consistent with equilibrium if and only if there exist $c^{h}(i) \in \mathbb{R}_{+}^{S}$ for all $h \in \mathcal{H}$ and all $i \in \mathcal{E}$ such that

(i) For each $h,\left(c^{h}(i), \rho(i)\right)_{i \in \mathcal{E}}$ satisfy the Afriat inequalities.

(ii) $(\phi \circ \rho(i)) \cdot\left(c^{h}(i)-e^{h}(i)\right)=0 \quad$ for all $i \in \mathcal{E}$ and all $h \in \mathcal{H}$.

(iii) $\sum_{h=1}^{H}\left(c^{h}(i)-e^{h}(i)\right)=0$ for all $i \in \mathcal{E}$.

The proof follows directly from Brown and Matzkin (1996). For prices to lie on an equilibrium correspondence, there have to exist budget-feasible individual consumptions that satisfy the Afriat inequalities which characterize choice compatible with utility maximization.

\section{Restrictions on counterfactual analysis}

As pointed out above, restrictions on counterfactual analysis can be divided into restrictions on beliefs and restrictions on probability-free prices $\rho$. The restrictions on beliefs are clear. Given any two observations on Arrow prices, $A(1), A(2)$, the largest eigenvalue and the eigenvector associated with these prices have to be the same. Furthermore, the largest eigenvalue has to be less than 1 . Theorem 1 shows that as far as probabilities and discounting are concerned these are the necessary and sufficient restrictions. The remaining question now concerns what we can say about the associated $\rho(1), \rho(2)$. We refer to restrictions on price changes always as restrictions on $\rho$ assuming that probabilities remain constant.

In this section we derive conditions on profiles of endowments which are necessary in order for price changes not to be arbitrary, as well as conditions that are sufficient for restrictions to exist. It is useful to distinguish two cases. We first assume that individual 
endowments change but aggregate endowments in each state $s \in \mathcal{S}$ remain constant across observations; then we move to the case that aggregate as well as individual endowments vary.

\subsection{Constant aggregate endowments}

Lemma 3 implies that for specified fixed aggregate endowments any finite set of distributions of individual endowments together with any prices are always consistent with equilibrium. This fact follows because we can choose each observation's individual consumption to be collinear to aggregate consumption. Generically the consumptions will all be different and strictly ordered. More formally, and without genericity assumptions, we have the following theorem:

Theorem 2 Suppose $N$ observations on individual endowments and prices $\left(\left(e^{h}(i)\right)\right.$, $\rho(i))_{i \in \mathcal{E}} \geq 0, \rho(i) \gg 0$ for all $i \in \mathcal{E}$, satisfy $\sum_{h} e_{s}^{h}(i)=\sum_{h} e_{s}^{h}(j)$ for all $s \in \mathcal{S}$ and all $i, j \in \mathcal{E}$. Then the observations are consistent with equilibrium, independently of $\phi \gg 0$.

Proof Given any $\phi \in \mathbb{R}_{++}^{S}$, for all $h \in \mathcal{H}$ and $i \in \mathcal{E}$ define consumption $c^{h}(i) \in \mathbb{R}_{++}^{S}$ by

$$
c^{h}(i)=\frac{(\phi \circ \rho(i)) \cdot e^{h}(i)}{(\phi \circ \rho(i)) \cdot e(i)} \cdot e(i)+\epsilon^{h}(i)
$$

with perturbations $\epsilon^{h}(i) \in \mathbb{R}^{S}$. These perturbations can be chosen arbitrarily small so that $c^{h}(i) \gg c^{h}(j)$ (or vice versa) for any $i \neq j$ while also satisfying $(\phi \circ \rho(i)) \cdot \epsilon^{h}(i)=$ 0 and $\sum_{h \in \mathcal{H}} \epsilon^{h}(i)=0$. (Note, there are only finitely many observations.) Now Condition (S) of Lemma 3 implies that the Afriat inequalities are satisfied for the constructed consumption vectors $\left(c^{h}(i)\right)_{i \in \mathcal{E}}$ and any arbitrary prices $(\rho(i))_{i \in \mathcal{E}} \gg 0$. Moreover, by construction markets clear and the budget constraints are satisfied. Thus, all three conditions of Lemma 4 are satisfied and the theorem follows.

Balasko and Tvede (2009a) derive the same result for an economy with general (i.e., not necessarily separable) utility. Our result is slightly stronger since theirs does not imply ours. Their method of proof (which is very different from ours) may also be used to derive our result. Balasko and Tvede reach the rather negative conclusion that 'finite collections of data that are not included in any equilibrium manifold make up a set that is certainly not large'. While this seems to suggest that for many global comparative statics exercises general equilibrium imposes no restrictions on observables, the crucial assumption in their analysis is that aggregate endowments remain constant. Therefore, their results (and our Theorem 2) are somewhat comparable to Mas-Colell (1977) who shows that without any variations in endowments, the equilibrium set can be arbitrary. 


\subsection{Changes in aggregate endowments}

Intuitively, clear restrictions should arise if aggregate endowments change. If, for example, aggregate endowments become more risky, prices in a representative agent economy become more volatile. One would expect that even with several heterogeneous agents, someone has to bear the risk and equilibrium prices must change accordingly. We first show that this intuition depends crucially on the values of individual endowments. One can always construct changes in individual endowments that destroy this intuition and lead to a situation where price changes can be arbitrary. Then we show that in the simple benchmark case of constant endowment shares across observations strong restrictions indeed do exist. In fact a much weaker assumption suffices to guarantee restrictions. We discuss this issue in detail below.

\subsubsection{The role of individual endowments}

When individual endowments are allowed to change arbitrarily together with aggregate endowments and if there are sufficiently many different agents, there are no restrictions on possible price changes. In other words, for given aggregate endowments (which may vary or be constant across observations) we can construct individual endowments such that there are no restrictions on associated prices. The following theorem formalizes this fact:

Theorem 3 Given aggregate endowments $(e(i))_{i \in \mathcal{E}} \gg 0$ and any $\phi \gg 0$, there always exist $H \geq N$ agents with individual endowments $\left(e^{h}(i)\right)_{i \in \mathcal{E}}^{h \in \mathcal{H}} \gg 0$ satisfying $\sum_{h} e^{h}(i)=e(i)$ such that any arbitrary positive prices $(\rho(i))_{i \in \mathcal{E}} \gg 0$ are consistent with equilibrium.

Proof Without loss of generality, we consider $H=N$. For sufficiently small $\epsilon>0$, let endowments of an individual $h$ in observation $i$ for each shock $s \in \mathcal{S}$ be as follows:

$$
e_{s}^{h}(i)= \begin{cases}i \epsilon+\delta e_{S}(i) & \text { if } i \neq h \\ e_{S}(i)-(H-1)\left(i \epsilon+\delta e_{s}(i)\right) & \text { otherwise }\end{cases}
$$

For sufficiently small $\epsilon>0$ and $\delta=0$, individual consumption $c^{h}(i)=e^{h}(i)$ is budget feasible, independently of prices. For each agent $h$ the consumption vectors are also strictly ordered and thus satisfy Condition (S) of Lemma 3. Therefore, for each agent the Afriat inequalities are satisfied, too, and all three conditions of Lemma 4 hold.

Note that the construction hinges critically on $H \geq N$. We do not know if a similar construction works with fewer agents. However, for a fixed number of agents we can always construct examples where price changes cannot be arbitrary.

Example 1 Consider $S=3, H=2$ and $e(1)=(10,1,1), e(2)=(1,10,1), e(3)=$ $(1,1,10)$. No matter what individual endowments, prices $\rho(1)=\rho(2)=\rho(3)$ cannot be rationalized. 
Note that one of the two agents must consume at least five units in the high-endowment state in (at least) two observations. W.l.o.g. suppose $c_{1}^{1}(1) \geq 5$ and $c_{2}^{1}(2) \geq 5$. Thus, $c_{2}^{1}(1)<c_{2}^{1}(2)$ but

$$
\frac{\rho_{2}(1) / \rho_{1}(1)}{\rho_{2}(2) / \rho_{1}(2)}=1
$$

and so Condition (N) of Lemma 3 is violated. That is, the first agent's consumption imposes restrictions on possible price changes.

This discussion points to one possible way how our initial intuition may fail. Changes in aggregate consumption might not be reflected in changes in individual consumption if at the same time incomes change in a way that the changes in aggregate consumption become irrelevant and individuals' consumption remains strictly ordered. This observation suggests that one possible way to obtain restrictions is to hold the fraction of individual endowments to aggregate endowments constant in each state across all observations, i.e., there are $\kappa_{s}^{h}>0, \sum_{h} \kappa_{s}^{h}=1$, such that $e_{s}^{h}(i)=\kappa_{s}^{h} e_{s}(i)$ for all observations $i=1, \ldots, N$. This assumption guarantees that there are prices for which in fact everybody will have higher consumption in shock $s$ for an observation where aggregate endowments in shock $s$ are higher. Note that this is a much weaker assumption than assuming collinear individual endowments-we return to this assumption in Sect. 5 below.

\subsubsection{Sufficient conditions for restrictions}

Constant endowment shares do ensure that there exist restrictions, but an assumption in the same spirit that is much weaker suffices. Price changes are restricted if there are two observations $i, j$ such that in some shock $s$ each individual's endowments increase (from observation $i$ to observation $j$ ) while in some other shock $s^{\prime}$ aggregate endowments weakly decrease (from $i$ to $j$ ). Note that the resulting change in aggregate endowments is also a necessary (and sufficient) condition for restrictions in the single agent case.

Theorem 4 Suppose there are two observations $i=1,2$ and shocks $s, s^{\prime} \in \mathcal{S}$ with $e_{s}^{h}(1)>e_{s}^{h}(2)$ for all agents $h \in \mathcal{H}$ and $e_{s^{\prime}}(1) \leq e_{s^{\prime}}(2)$. Then prices cannot be arbitrary.

Proof Given profiles of endowments and any $\phi \gg 0$, we show that there exist price vectors $\rho(1), \rho(2) \gg 0$ that are not consistent with equilibrium. We normalize $\rho_{s^{\prime}}(i)=1$ for both observations. Because $e_{s}^{h}(1)>e_{s}^{h}(2)$ we can choose $\epsilon, \delta>0$ such that $e_{s}^{h}(1)-\epsilon>e_{s}^{h}(2)+\delta$. Next we can choose prices $\rho_{S}(2)<\rho_{S}(1)$ sufficiently large such that for each agent $h$, the budget constraints

$$
(\phi \circ \rho(i)) \cdot c^{h}(i) \leq(\phi \circ \rho(i)) \cdot e^{h}(i)
$$

yield the following implications: If for an agent $h$ it holds that $c_{s}^{h}(1) \leq e_{s}^{h}(1)-\epsilon$ then it follows that $c_{s^{\prime}}^{h}(1)>e_{s^{\prime}}(1)$. Similarly, if for an agent $h$ it holds that $c_{s}^{h}(2) \geq e_{s}^{h}(2)+\delta$ 
then it follows that $c_{s^{\prime}}^{h}(2)<0$. Market clearing and the non-negativity constraints on consumption thus ensure $c_{s}^{h}(1)>c_{s}^{h}(2)$ for all agents $h \in \mathcal{H}$. Market clearing also implies for at least one agent $h$ that $c_{s^{\prime}}^{h}(1) \leq c_{s^{\prime}}^{h}(2)$. But by construction the prices $\rho_{s}(1)>\rho_{s}(2)$ satisfy $\frac{\rho_{s^{\prime}}(1) / \rho_{s}(1)}{\rho_{s^{\prime}}(2) / \rho_{s}(2)}=\frac{1 / \rho_{s}(1)}{1 / \rho_{s}(2)}<1$ and so Condition (N) of Lemma 3 is not satisfied. The Afriat inequalities cannot hold.

The theorem may at first appear to contradict Balasko and Tvede (2009a) claim that their result on the absence of restrictions can be extended to an open neighborhood of endowments. While our result does require the strong assumption of separable utility [made neither in Balasko and Tvede (2009a) nor in Balasko and Tvede (2009b)], this is not the main difference. Balasko and Tvede (2009a) show that for given profiles of endowments and prices there is always a small open neighborhood for which the observations are still consistent with equilibrium. We show that if endowments satisfy the inequalities in Theorem 4, no matter how small the differences across observations, there always exist prices that are inconsistent with equilibrium.

\section{Counterfactual analysis and the representative agent}

The analysis in the previous section reveals the key role of the interplay between aggregate and individual endowments. This observation naturally motivates us to compare the restrictions imposed by models with heterogeneous agents and models with a representative agent. Naturally, aggregate endowments in the heterogeneous-agents model are the corresponding endowments of the representative agent. Since we do not confine ourselves to any specific type of felicity functions, we do not need to be concerned about the aggregation of preferences (see Hara et al. (2007) and Hara (2008) for recent results on this topic).

We now examine the possible relationships between restrictions in models with a heterogeneous agent and restrictions in models with a representative agent. As a first step we show that restrictions from a representative-agent model carry over to a model with multiple agents if we choose the endowments of one agent sufficiently large and the endowments of all other agents very small. This observation is the content of the following result:

Theorem 5 For given aggregate endowments $(e(i))_{i \in \mathcal{E}}$, all different across observations, and prices that are inconsistent with a representative agent there always exist individual endowments $\left(e^{h}(i)\right)^{h \in \mathcal{H}} \gg 0, \sum_{h} e^{h}(i)=e(i)$ such that these prices cannot be equilibrium prices.

Proof For $\epsilon>0$, take $e_{s}^{h}(i)=\epsilon$ for all $h=1, \ldots, H-1$, all $i$ and all $s$. Since consumer $H$ has to consume on his budget set, eventually, for sufficiently small $\epsilon$, his consumption is arbitrary close to aggregate endowments and hence if prices and choices are inconsistent for aggregate endowments, they must be inconsistent for the representative agent, as well as for agent $H$ whose consumption can be forced to be arbitrarily close to the representative agent's consumption.

The proof of the theorem relies on very extreme endowments. This feature is only a sufficient condition. We can easily construct an example where for a large set of 
individual endowments the counterfactual analysis for a representative agent economy has also to hold in an economy with several agents.

Example 2 Consider $N=2, H=2, S=2$ and suppose that $\phi=(1,1), \rho(1)=$ $(1,1), e(1)=(3,1)$ and $e(2)=(1,3)$. Also suppose that $e_{1}^{1}(1)+e_{2}^{1}(1)=3$ and $e_{1}^{1}(2)+e_{2}^{1}(2)<3$. Then it is impossible that prices and endowments in the second observation satisfy

$$
\rho_{1}(2) e_{1}^{1}(2)+\rho_{2}(2) e_{2}^{1}(2)>2 \rho_{1}(2)+\rho_{2}(2)
$$

and

$$
\rho_{1}(2)+2 \rho_{2}(2)>\rho_{1}(2) e_{1}^{1}(2)+\rho_{2}(2) e_{2}^{1}(2) .
$$

In observation 1 agent 1 consumes somewhere on his budget line that connects the points $(3,0)$ and $(2,1)$ in the Edgeworth box. If $\rho_{1}(2) e_{1}^{1}(2)+\rho_{2}(2) e_{2}^{1}(2)>2 \rho_{1}(2)+$ $\rho_{2}(2)$ then agent 1 can afford any consumption in observation 2 that he could have afforded in observation 1 and that lies in the Edgeworth box for observation 1. The weak axiom of revealed preferences then implies that at prices and incomes of observation 1 , the agent cannot afford the consumption he chose at observation 2 . Since in observation 2 he must choose somewhere on his budget line in the Edgeworth box, it is certainly impossible that at prices and income of observation 1 he could afford everything in observation 2's Edgeworth box that he can afford under income and prices of observation 2. So it is impossible that the two budget lines cross outside of both of the two Edgeworth boxes, i.e., that $\rho_{1}(2)+2 \rho_{2}(2)>\rho_{1}(2) e_{1}^{1}(2)+\rho_{2}(2) e_{2}^{1}(2)$.

The restrictions in Example 2 follow from restrictions on agent 1's choices. Yet, this agent seems far from a 'dominating' agent whose consumption must be close to aggregate endowments.

\subsection{More or fewer restrictions through heterogeneity?}

Theorem 5 and Example 2 show that it is certainly possible for the restrictions from a representative-agent economy to carry over to the heterogeneous agents economy. We next show that such a relationship does not hold in general. In fact, anything can happen, the heterogeneous-agent economy may impose an altogether different set of restrictions than the corresponding representative-agent economy.

We can easily construct an example of a representative-agent economy that imposes restrictions. Theorem 3 then readily implies that we can construct a corresponding heterogeneous-agents economy without any restrictions.

The converse relationship is also possible, that is, there are no restrictions for the representative agent but for appropriately chosen individual endowments the heterogeneous-agents economy imposes restrictions. Suppose $e(i) \gg e(i+1)$ for all $i=1, \ldots, N-1$. Condition (S) of Lemma 3 implies that price changes can be arbitrary for a representative agent. With heterogeneous agents this is false. The following example proves this fact: 
Example 3 Consider $S=2$ states with $N=3$ observations with aggregate endowments of $e(1)=(1,1), e(2)=(1.1,10), e(3)=(1.2,100)$ and suppose $\phi=(1,1)$. For $H=2$ agents with $e^{1}(1)=(1-\epsilon, 1-\epsilon), e^{1}(2)=(1 / 2,1)$, and $e^{1}(3)=(0.9,50)$ the prices $\rho(1)=\rho(2)=\rho(3)=(1,1 / 1000)$ are inconsistent with equilibrium if $\epsilon>0$ is sufficiently small.

Condition (N) of Lemma 3 implies that for prices to remain constant across observations consumptions must be strictly ordered. However, the following argument shows that it is impossible that both agents' consumptions are ordered across observations. If $c^{1}(1)$ and $c^{1}(2)$ are ordered then budget feasibility for agent 1 combined with market clearing implies $c^{1}(1) \gg c^{1}(2)$. By the same argument, for sufficiently small $\epsilon$, it is impossible that $c^{1}(3) \gg c^{1}(1)$. But if $c^{1}(1) \gg c^{1}(3)$ and $c^{1}(1) \gg c^{1}(2)$, it is impossible that for agent $2 c^{2}(2)$ and $c^{2}(3)$ are ordered. Agent 1 consumes less than 1 in shock 2 in both observation 2 and 3 and so market clearing implies $c_{2}^{2}(3)>c_{2}^{2}(2)$. But since agent 1 consumes close to 0.9 in shock 1 in observation 3 and close to 0.5 in observation 2, market clearing must imply that $c_{1}^{2}(3)<c_{1}^{2}(2)$.

The example shows that agent heterogeneity can, given particular individual endowments, impose additional restrictions on global comparative statics. Of course, for this to happen it is crucial that the distribution of endowments changes together with aggregate endowments.

Observe that the construction in the example needs $N \geq 3$ observations. For $N=2$ observations no such example exists; instead, if aggregate endowments and prices are consistent with a representative agent then they must be consistent with a heterogeneous-agent economy for any individual endowments. With only two observations we can always construct individual consumptions to be strictly ordered so that Condition (S) of Lemma 3 is satisfied.

We summarize our comparison of representative-agent and heterogeneous-agents economies in the following theorem:

Theorem 6 Restrictions in the representative agent economy are neither necessary nor (for $N \geq 3$ ) sufficient for restrictions in the heterogeneous agents economy.

Proof Theorem 3 implies that for a representative agent economy with restrictions we can always construct a heterogeneous agents economy without restrictions. Theorem 5 implies that for a representative agent economy with restrictions we can always construct a heterogeneous agents economy having the same restrictions. Example 3 shows a representative agent economy without restrictions and a corresponding heterogeneous agents economy with restrictions.

\subsection{Collinear endowments}

It is difficult to find general conditions on individual endowments that imply that representative agent restrictions carry over to the economy with several agents. As an illustration, we consider the simple (but for some applications relevant) case of collinear endowments. Theorem 4 states that there are restrictions for the heterogeneous-agent economy whenever there are restrictions for the representative-agent 
economy: For the presence of restrictions aggregate endowments have to increase in one state and weakly decrease in another. Since individual endowments are a fixed fraction of aggregate endowments, the condition of the theorem holds.

Note that the representative agent conditions on prices are sufficient in this case, since each agent can simply consume his endowments. However, the following example shows that while price changes cannot be arbitrary, counter-intuitive price changes that are impossible for the single agent economy are now possible.

Example 4 Suppose $H=2, S=2, N=2$ with $e^{1}(1)=e^{2}(1)=(1,2), e^{1}(2)=$ $e^{2}(2)=(1,2.01)$ and $\phi=(1,1)$. Prices $\rho(1)=(1,0.5)$ and $\rho(2)=(1,0.6)$ are possible in the heterogeneous agent economy, although endowments are identical and these prices are impossible in the representative agent model.

We show that the three conditions of Lemma 4 are satisfied. Let the two agents' consumption vectors be $c^{1}(1)=(1.3,1.4), c^{2}(1)=(0.7,2.6)$ and $c^{1}(2)=(1.31,2.01-$ $0.31 / 0.6), c^{2}(2)=(0.69,2.01+0.31 / 0.6)$. These consumption vectors satisfy the market clearing conditions and budget equations. Condition (S) of Lemma 3 also holds since $c^{1}(1)<<c^{1}(2)$ and $c^{2}(1)>>c^{2}(2)$. Thus, the Afriat inequalities hold.

Now consider the representative agent economy with consumptions and endowments $c(1)=e(1)=(2,4)$ and $c(2)=e(2)=(2,4.02)$. Since $c_{2}(2)>c_{2}(1)$ and $c_{1}(2)=c_{1}(1)$ Condition $(\mathrm{N})$ of Lemma 3 is violated,

$$
\frac{\rho_{1}(2) / \rho_{2}(2)}{\rho_{1}(1) / \rho_{2}(1)}=\frac{1 / 0.6}{1 / 0.5}<1
$$

and so the Afriat inequalities do not hold.

The reason why the representative agent intuition does not carry over to a model with different tastes is as follows. If one agent is close to risk neutral, an increase in the price of a shock with large aggregate endowments results in a decrease of the agent's consumption in both shocks, the more risk-averse agent's consumption increases in both shocks and the resulting aggregate consumption can increase in the shock where the price increased.

\section{Conclusion}

In this paper, we examine the possible equilibrium price changes that can result from changes in individual endowments in the Lucas (1978) asset pricing model with dynamically complete markets. We perform this analysis under the assumption of time-separable preferences, an assumption that is almost standard in general equilibrium models in macroeconomics, finance, and public finance. We make no other assumptions, in particular no assumptions on specific parametric specifications of the utility functions. Our results thus apply to a large class of economically interesting models.

We establish three main results. First, we show that equilibrium price changes can be arbitrary if individual endowments vary but aggregate endowments are held fixed. Second, we show that changes in aggregate endowments always lead to restrictions on possible price changes, if in at least one state aggregate endowments weakly decrease 
while in some other state all individuals' endowments increase. Last, we show that restrictions from the representative-agent version of the model are neither necessary nor sufficient for restrictions in a heterogeneous agents economy.

Despite our mild assumptions, which hold in many instances of counterfactual policy analysis in economic practice, we are able to show that restrictions on equilibrium prices do exist. This paper, therefore, serves as a general framework for future research into the exact nature of these restrictions. Our results suggest that a more specific description of possible restrictions will require much stronger assumptions on the economic model.

\section{References}

Afriat, S.: The construction of a utility function from demand data. Int Econ Rev 8, 67-77 (1967)

Balasko, Y., Tvede, M.: The geometry of finite equilibrium datasets. J Math Econ (2009a, forthcoming)

Balasko, Y., Tvede, M.: Individual preference rankings compatible with prices, income distributions and total resources. Econ Theory (2009b, forthcoming)

Bewley, T.: Existence of equilibria in economies with infinitely many commodities. J Econ Theory 43, 514-540 (1972)

Brown, D.J., Matzkin, R.: Testable restrictions on the equilibrium manifold. Econometrica 64, 1249-1262 (1996)

Constantinides, G.: Intertemporal asset pricing with heterogeneous consumers and without demand aggregation. J Bus 55, 253-267 (1982)

Cuoco, D., Zapatero, F.: On the recoverability of preferences and beliefs. Rev Financ Stud 13, 417-431 (2000)

Danthine, J.-P., Donaldson, J.B., Giannikos, C., Guirguis, H.: On the consequences of state dependent preferences for the pricing of financial assets. Financ Res Lett 1, 143-153 (2004)

Duffie, D.: Security Markets: Stochastic Models. London: Academic Press (1988)

Dumas, B., Uppal, R., Wang, T.: Efficient intertemporal allocations with recursive utility. J Econ Theory 93, 240-259 (2000)

Hara, C.: Aggregation of state-dependent utilities, working paper. Institute of Economic Research, Kyoto University (2008)

Hara, C., Huang, J., Kuzmics, C.: Representative consumer's risk aversion and efficient risk-sharing rules. J Econ Theory 137, 652-672 (2007)

Horn, R., Johnson, C.R.: Matrix Analysis. London: Cambridge University Press (1985)

Kubler, F.: Observable restriction of general equilibrium with financial markets. J Econ Theory 110, 137153 (2003)

Kubler, F., Schmedders, K.: Generic inefficiency of equilibria in the general equilibrium model with incomplete asset markets and infinite time. Econ Theory 22, 1-15 (2003)

Kubler, F., Schmedders, K.: Non-parametric counterfactual analysis in dynamic general equilibrium. PIER Working Paper 07-027 (2007)

Lucas, R.E. Jr.: Asset prices in an exchange economy. Econometrica 46, 1429-1445 (1978)

Mas-Colell, A.: On the equilibrium price set of an exchange economy. J Math Econ 4, 117-126 (1977)

Melino, A., Yang, A.: State-dependent preferences can explain the equity premium puzzle. Rev Econ Dyn 6, 806-830 (2003)

Rockafellar, R.T.: Convex Analysis. Princeton: Princeton University Press (1970)

Varian, H.: Non-parametric tests of consumer behaviour. Rev Econ Stud 50, 99-110 (1983)

Varian, H.: Non-parametric tests of models of investor behavior. J Financ Quant Anal 18, 269-278 (1983)

Wang, S.: The integrability problem of asset prices. J Econ Theory 59, 199-213 (1993) 